Research Paper

\title{
Taurine Transporter Regulates Adipogenic Differentiation of Human Adipose-Derived Stem Cells through Affecting Wnt/ $\beta$-catenin Signaling Pathway
}

Xiaodan Hou ${ }^{*}$, Zhixue Wang ${ }^{*}$, Fang Ding 1 , Yang He${ }^{1}$, Pengyuan Wang ${ }^{3}$, Xia $\mathrm{Liu}^{3}$, Feng Xu${ }^{4}$, Jun Wang ${ }^{4 凶}$, Yili Yang $1^{\bowtie}$

1. Suzhou Institute of Systems Medicine, Center of Systems Medicine, Chinese Academy of Medical Sciences, Suzhou215123, Jiangsu, China

2. Department of Burn and Plastic Surgery, North District of Suzhou Municipal Hospital, Suzhou 215008, Jiangsu, China

3. Department of Pharmacology, College of Pharmacy, Second Military Medical University, Shanghai 200433, China

4. Department of Emergency, The First Affiliated Hospital of Soochow University, Suzhou, 215006, Jiangsu Province, China

*These authors contributed equality.

$\triangle$ Corresponding authors: Yili Yang, Suzhou Institute of Systems Medicine, Center of Systems Medicine, Chinese Academy of Medical Sciences, Suzhou215123, Jiang su, China. Email: yyl@ism.cams.cn. and Jun Wang, Department of Emergency, The First Affiliated Hospital of Soochow University, Suzhou, 215006, Jiangsu Province, China. Email: wangjun_3236@suda.edu.cn.

(C) Ivyspring International Publisher. This is an open access article distributed under the terms of the Creative Commons Attribution (CC BY-NC) license (https://creativecommons.org/licenses/by-nc/4.0/). See http://ivyspring.com/terms for full terms and conditions.

Received: 2018.11.26; Accepted: 2019.02.16; Published: 2019.04.22

\begin{abstract}
Increased adipocytes are associated with obesity and many human disorders including cancers. To further understand the molecular mechanisms of adipogenesis, transcriptome sequencing was performed to find genes involved in the adipogenic differentiation of human adipose-derived stem cells (hASCs). The mRNA of taurine transporter (TauT, also known as SLC6A6) was found significantly upregulated in hASCs undergoing differentiation. TauT expression was also markedly increased in fat tissues from obese mice induced by high fat diet or genetic mutations (ob/ob and $\mathrm{db} / \mathrm{db}$ mice). In vitro, downregulation of TauT attenuated effectively the adipogenic differentiation of hASCs, and TauT overexpression promoted the formation of adipocytes. Among the molecules transported by TauT, hypotaurine and $\beta$-alanine promoted adipocyte formation, whereas taurine inhibited the process. Moreover, the inhibitory effect of TauT knockdown on hASCs differentiation was largely reversed by hypotaurine and $\beta$-alanine through promoting the downregulation of $\beta$-catenin. These results indicated that TauT regulate adipocyte formation through transported amino acids and may serve as a target for therapeutic intervention of obesity.
\end{abstract}

Key words: human adipose-derived stem cells, taurine transporter, adipogenic differentiation

\section{Introduction}

In addition to provide thermal insulation and protective padding, and to store excess glucose in the form of triglycerides, adipose tissue has been increasingly recognized as an active endocrine organ 1 . It secretes a number of hormone-like adipokines that play essential roles in energy balance and homeostasis of glucose, lipids, and systemic inflammation 2 , 3 . Noteworthily, the predominantly adipose-produced leptin acts on receptor in the arcuate nucleus of the hypothalamus to inhibit food intake ${ }^{4}$. Dysfunction of leptin or its receptor results in obesity in mice $(o b / o b$ and $d b / d b$ mice) and human, whereas most of the obese individuals have increased leptin levels and develop leptin resistance 5 .

In contrast to most tissues, adipose tissue exhibits uniquely an almost unlimited capacity to expand. Overweight and obesity, manifested by abnormal or excessive adiposity that are usually defined by body mass index, has been recognized as a global epidemic since the end of last century ${ }^{6}$. Obesity is often associated with altered steroid hormone and adipokines production, insulin resistance, dyslipidemia, and subclinical chronic inflammation?, and contributes to cancer initiation, progression, and 
metastasis - Although the mechanisms linking increased adiposity to malignancy are incompletely understood, it is evident that adipose tissue-derived cytokines and adipose cells in tumors all play important roles in cancer development.

The excessive adiposity in adipose tissue results from increased adipocyte number, adipocyte hypertrophy, and metabolic dysfunction?. It has been shown that precursors of adipocytes, often designed as adipose-derived stem cells (ASCs), are capable of differentiating into adipocytes, and presumably play a critical role in the increase and dysfunction of adipocytes in obese individuals $\underline{10}$. To explore the molecular mechanisms involved in adipocyte differentiation, we isolated hASCs from subcutaneous adipose tissues and performed transcriptome sequencing. Taurine transporter (TauT, also known as SLC6A6) was found significantly increased during the early stage of adipogenisis and in fat tissues from obese mice. TauT transported molecules hypotaurine and $\beta$-alanine promoted adipogenic differentiation, whereas taurine and TauT knockdown impeded adipogenic differentiation of hASCs in culture. Further analysis revealed that TauT appears required for the downregulation of $\beta$-catenin during adipocyte formation. Thus, the taurine transporter plays an important role in adipogenesis, and may serves as a target for therapeutic intervention of obesity.

\section{Materials and Methods}

\section{Isolation of human adipose-derived stem cells}

This study was approved by the Ethical Review Board of the First affiliated hospital of Soochow University. Human adipose tissue was collected from patients aged 20 to 35 years old. After surgery, the adipose tissues were immediately transported to the laboratory in sterile PBS buffer containing $100 \mathrm{U} / \mathrm{ml}$ penicillin and $100 \mu \mathrm{g} / \mathrm{ml}$ streptomycin on ice. After $2 x$ washes, the adipose tissues were minced using sterile scalpels and scissors and then digested with $0.1 \%$ type I collagenase in a shaking water bath at $37{ }^{\circ} \mathrm{C}$ for 30 min. After digestion, the tissue was filtered with a $70 \mu \mathrm{m}$-mesh sieve, and the filtrate was centrifuged at $1000 \times \mathrm{g}$ for $10 \mathrm{~min}$. Cells obtained from the pellet were cultured with DMEM/F-12 containing 10\% FBS. These cells were identified as $\mathrm{CD} 34-/ \mathrm{CD}^{+} 3^{+} / \mathrm{CD} 105^{+}$ by flow cytometry. After 3 passages, the cells were frozen in liquid nitrogen for further experiments.

\section{Cell culture and adipogenic differentiation}

Human adipose-derived stem cells (hASCs) medium consisted of DMEM/F-12 Medium with 10\% FBS, $100 \mathrm{U} / \mathrm{ml}$ penicillin and $100 \mu \mathrm{g} / \mathrm{ml}$ streptomycin at $37^{\circ} \mathrm{C}$ in a humidified $5 \% \mathrm{CO} 2,95 \%$ air atmosphere. For experiments, cells were seeded and grown to confluence for 2 days. Adipocyte differentiation was induced by the pro-adipogenic components Insulin $(10 \mathrm{nM})$, rosiglitazone $(100 \mathrm{nM}), 3$-isobutyl-1methylxantine (IBMX, $100 \mu \mathrm{M})$ and dexamethasone (Dex, $1 \mu \mathrm{M}$ ) for 3 days. Cells were then switched to adipogenesis progression inducer Insulin $(10 \mathrm{nM})$, rosiglitazone $(100 \mathrm{nM})$, and dexamethasone (Dex, 1 $\mu \mathrm{M})$ for 2 days. Finally, cells were maintained with Insulin $(10 \mathrm{nM})$.

\section{Plasmid construction and siRNA transfection}

To construct the pLVX- TauT plasmid, a fragment of the TauT cDNA (GenBank accession number: NM_003043.5) was amplified and cloned into the lentivirus vector pLVX. TauT siRNAs sequence: siRNA\#2 sense: 5-GCUAUGCCUCCGUUGUAAUTT -3, antisense: 5- AUUACAACGGAGGCAUAGCTT-3; siRNA\#3 sense: 5-GGAACACACCUCACUGCAT-3, antisense: 5-AUGCAGUGAGGUGUGUUCCTT-3. The TauT ectopic-expression plasmid and siRNAs were transfected into hASCs every 3 days.

\section{Analysis of lipid accumulation}

Staining of intracellular lipids by Oil Red $\mathrm{O}$ was performed as described previously $\underline{11}$. To quantify intracellular triglyceride accumulation, cells were washed with PBS twice. In order to obtain quantitative data, $500 \mu$ l of isopropyl alcohol was added to the stained culture dish. After 5 minutes, the absorbance of the extract was assayed by a spectrophotometer at $510 \mathrm{~nm}$.

\section{Animal experiments}

Twelve-week-old wild type, $o b / o b$ and $d b / d b$ mice were purchased from Shanghai Laboratory Animal Center. The animals were operated according to the protocol approved by the Institutional Animal Care and Use Committee of the Suzhou Institute of Systems Medicine. Eight-week-old mice were fed with high fat diet for 2 months. Mice were sacrificed and subcutaneous and visceral mouse fats tissues were excised and extracted for total RNA.

\section{RNA extraction and real-time PCR}

The culture medium was removed, and the cells were immediately washed with ice-cold PBS. Subsequently, $1 \mathrm{ml}$ of TRIzol reagent was added, and total cellular RNA was extracted using the acid guanidinium thiocyanate-phenol-chloroform method. Total RNA $(1 \mu \mathrm{g})$ was used as a template for an MMLV-RT reverse transcriptase reaction, which was performed according to the manufacturer's instructtions. Real-time quantitative reactions were set up in triplicate in a 96-well plate, and each reaction contained $1 \mu \mathrm{l}$ of cDNA and the SYBR Green PCR mix, to which gene-specific forward and reverse PCR 
primers were added. Melting curves were analysed to verify the specificity of the RT-PCR reaction and the absence of primer dimer formation. The following primers were used: PPARY sense: 5-TCGCTGATGCA CTGCCTATG-3, antisense: 5-GAGAGGTCCACAGA GCTGATT-3; and FABP4 sense: 5-GGGCCAGGAATT TGACGAAG-3, antisense: 5-CGCATTCCACCACCA GTTTATC-3; $\beta$-actin sense: 5 -GCGGGAAATCGTGC GTGACATT-3, antisense: 5-GATGGAGTTGAAGGT AGTTTCG-3; human TauT sense: 5-TTTTGTGTCTGG CTTCGCAATTT-3, antisense: 5-TGGGTAGGCAATG AAGGCCAG-3; mouse TauT sense: 5-GCACACGGC CTGAAGATGA-3, antisense: 5-ATTTTTGTAGCAGA GGTACGGG-3. The mRNA levels of the target genes were normalized to $\beta$-actin. Each target was measured in triplicate, and data were analyzed using GraphPad Prism 5.

\section{RNAseq}

hASCs adipogenic differentiation was induced by the pro-adipogenic components for 3 days. Total RNA of the control and induced samples was extracted using TRIzol reagent. $1 \mu \mathrm{g}$ total RNA with RIN value above 7 was used for following library preparation. Next generation sequencing library preparations were constructed according to the manufacturer's protocol (NEBNext巴) Ultra ${ }^{\mathrm{TM}}$ RNA Library Prep Kit for Illumina $\left.{ }^{\circledR}\right)$. The sequences were processed and analyzed by GENEWIZ.

\section{Immunoblotting}

The cells were immediately placed on ice and washed with ice-cold PBS. Total protein extract was prepared with RIPA lysis buffer $(25 \mathrm{mM}$ Tris- $\mathrm{HCl}$ at $\mathrm{pH}$ 7.5, $2 \mathrm{mM}$ EDTA, $25 \mathrm{mM} \mathrm{NaF}$ and 1\% Triton $X-100)$ containing $1 \mathrm{X}$ protease inhibitor mixture (Roche) and $1 \times$ PMSF. The proteins were resolved on $8-12 \%$ SDS-polyacrylamide gels and transferred to polyvinylidene fluoride membranes (Merck Millipore). The membranes were blocked with $5 \%$ nonfat milk in TBST for 1 hour. Proteins of interest were detected with specific antibodies, blots were scanned using a ChemiDoc ${ }^{\mathrm{TM}}$ MP imaging system (Bio-Rad).

\section{Reagents and antibodies}

TRIzol reagent was purchased from Invitrogen. Fast-start universal SYBR Green master mix was from Roche. The inhibitor TWS119 was from Selleck. The antibodies used for immunoblotting (IB): PPARY (Cell Signaling, \#2443), FABP4 (Cell Signaling, \#3544), TauT (Absin, abs140562a), $\beta$-catenin (Cell Signaling, \#8480), $\beta$-actin (Santa Cruz, sc-47778), GAPDH (Proteintech, 60004-1-Ig), a-Tubulin (Santa Cruz, sc-58667).

\section{Statistical Analysis}

The data are presented as the means \pm SD. All data are representative of at least three independent experiments. The differences between groups were assessed by Student's t-test; all reported differences are ${ }^{*} p<0.05,{ }^{* *} p<0.01,{ }^{* * *} p<0.001$ unless otherwise stated.

\section{Results}

\section{Upregulation of taurine transporter in adipogenic differentiation}

To explore the molecular mechanisms of adipocyte differentiation, we isolated and cultured human adipose-derived stem cells (hASCs) from subcutaneous adipose tissues of donors undergoing plastic surgeries. Transcriptome sequencing was performed to compare the expression of genes in hASCs induced to undergo adipocyte differentiation for 3 days with those of uninduced. The induction changed the gene expression profile markedly (Figure 1A). While the levels of 216 genes were notably downregulated, the expressions of 133 genes were upregulated more than 2-folds (Table S1). Among the elevated genes was SLC6A6, which was ubiquitously expressed in many tissues and encodes a sodium and chloride-ion dependent transporter for taurine and $\beta$-alanine, often referred as taurine transporter (TauT). RT-PCR and immunoblotting analysis showed that TauT mRNA and protein levels increased significantly in hASCs after a 3-day induction, which was accompanied by the increase of fatty acid binding protein 4 (FABP4), a well-known marker for adipogenic differentiation (Figure 1B \& C).

The $o b / o b$ and $d b / d b$ mice harbor mutations in leptin and its receptor and become profoundly obese $\underline{12}$. Variations in the leptin receptor have also been associated with obesity in human and with increased susceptibility to Entamoeba histolytica infections $\underline{13}$. We therefore examined the expression of TauT in fat tissues from the $o b / o b$ and $d b / d b$ mice. Compared with wild type littermates, both obese mice had significantly increased levels of TauT mRNA (Figure 1D\&E). Moreover, both subcutaneous and visceral fats from mice fed with high fat diet also expressed elevated levels of TauT mRNA (Figure 1F\&G). Therefore, TauT might play an important role in adipogenesis and in the formation of obesity.

\section{TauT promoted the adipogenic differentiation of hASCs}

To determine whether the increased TauT expression is important in adipogenic differentiation, use was made of siRNA to knockdown TauT in hASCs induced to differentiate into adipocyte. As shown in Figure 2A, transfection of specific siRNA in induced hASCs markedly reduced the levels of TauT 
mRNA and protein. As such, the expression of adipogenic differentiation marker FABP4 and PPARY was also downregulated by Day 3 (Figure 2A \&B). Noteworthily, PPARY induction appeared relative early in the differentiation and acted as a master transcription factor to induce the expression of downstream targets including FABP4, a marker for later stage of differentiation. Furthermore, the
A

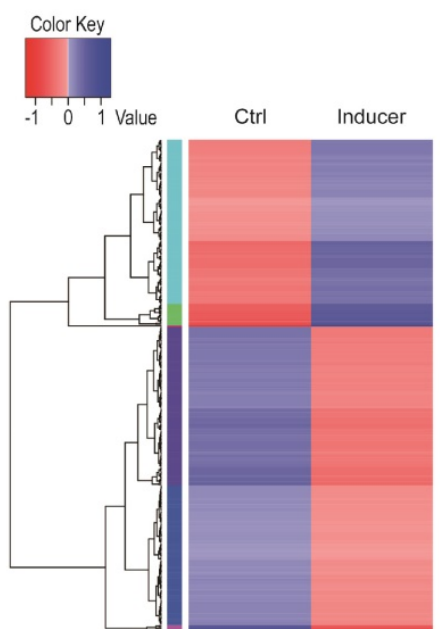

D

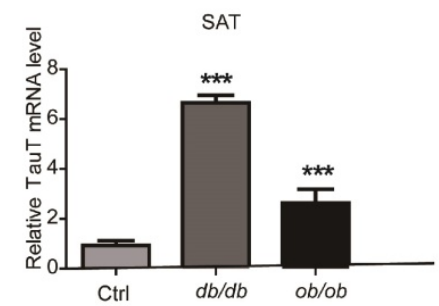

$\mathbf{F}$

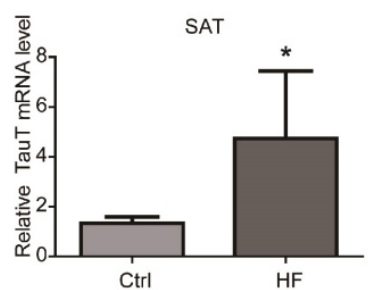

B

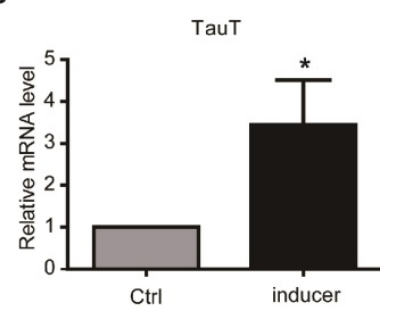

C

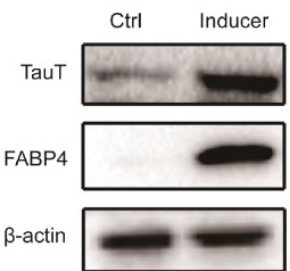

E

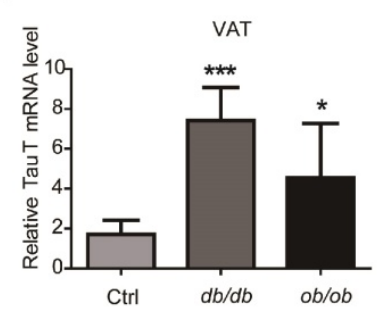

G

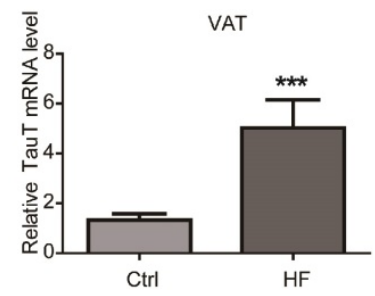

Figure 1. Upregulation of taurine transporter in adipogenic differentiation. A. Transcriptome sequencing of hASCs treated with or without adipogenic inducer. Gene expression profiles were performed with $\log 10(\mathrm{RPKM}+1)$ value, with high expression gene in blue and low expression gene in red. Color from red to blue indicates higher gene expression. B. The mRNA expression of TauT in the presence or absence of inducer. hASCs were induced by the adipogenic components for 3 days and mRNA levels were analysed by quantitative PCR and normalised to $\beta$-actin $\left(n=3,{ }^{*} p<0.05\right)$. C. The protein expression of TauT in the presence or absence of inducer. hASCs were induced by the adipogenic components for 3 days. FABP4 were detected as adipogenic differentiation marker. D. The mRNA levels of TauT in subcutaneous fat tissues of ob/ob or $\mathrm{db} / \mathrm{db}$ mice. Mice at the age of 12 weeks and age-matched wild type were excised and extracted for RNA $(n=5, * * * p<0.001)$. E. The mRNA levels of TauT in visceral fat tissues of ob/ob or $\mathrm{db} / \mathrm{db}$ mice. Mice at the age of 12 weeks and age-matched WT controls were excised and extracted for RNA $(n=5, * p<0.05, * * * p<0.001)$. F. The mRNA levels of TauT in subcutaneous fat tissues of high-fat fed mice. Mice at the age of 8 weeks were fed with high fat diet for 2 months. Then mice were excised and extracted for RNA $(n=5, * * * p<0.05)$ G. The mRNA levels of TauT in visceral fat tissues of high-fat fed mice. Mice at the age of 8 weeks were fed with high fat diet for 2 months. Then RNA was extracted from the tissues $(n=5, * * * p<0.001)$. All Western blots were independently repeated three times, and RT-PCR data are presented as the means \pm SD.
TauT-specific siRNA markedly reduced the formation of adipocytes by Day 9, as measured by Oil Red O staining (Figure 2C) and triglyceride quantification (Figure 2D).

We also transfected hASCs cells with TauTexpressing plasmid and induced them toward adipocytes. Compared these transfected with control construct, the induction of FABP4 and PPARY in TauT-expressing hASCs were markedly enhanced at both mRNA and protein levels by Day 3 (Figure 2E\&F). Moreover, enforced TauT expression promoted the formation of adipocytes as measured by Oil Red O staining at Day 9 (Figure 2G) and triglyceride quantification (Figure 2H). Thus, increased level of TauT appeared promote adipogenesis and is required for differentiation toward adipocytess under these conditions.

\section{Taurine, Hypotaurine and $\beta$-alanine modulated the adipogenic differentiation of hASCs}

TauT (SLC6A6) is a member of the sodium and chloride-ion dependent transporters family that transports taurine, hypotaurine and $\beta$-alanine $\underline{14}$. The involvement of TauT in adipogenic differentiation prompted us to examine the effects of these amino acids on the process in hASCs. As assessed by RT-qPCR and immunoblotting, administration of hypotaurine to the culture significantly increased the expression of differentiation markers FABP4 and PPARY by day 3 in induced hASCs (Figure 3A). The effects were dose-dependent and significant in the range of $5 \mu \mathrm{M}-5 \mathrm{mM}$. Similar changes of FABP4 and PPAR $\gamma$ were found after the induced cells were treated with $\beta$-alanine (Figure 3B). In contrast, the levels of FABP4 and PPARy were markedly decreased when the induced hASCs were exposed to taurine (Figure 3C). The inhibitions were also significant dose-dependently in the range of $50 \mu \mathrm{M}-5 \mathrm{mM}$, although taurine as well as hypotaurine and $\beta$-alanine did not affect notably the growth of hASCs (data not shown). The effects of these amino acids on induced hASCs were further evaluated by Oil Red O staining and triglyceride quantification. As shown in Figure 3D, hypotaurine and $\beta$-alanine treatment led to markedly increase intracellular lipid accumulation by day 9 compared to the control, whereas the addition of taurine resulted in decrease of the Oil Red O staining. Therefore, the substrates of TauT, hypotaurine, taurine and $\beta$-alanine all modulate the adipogenic differentiation of hASCs effectively. 
A

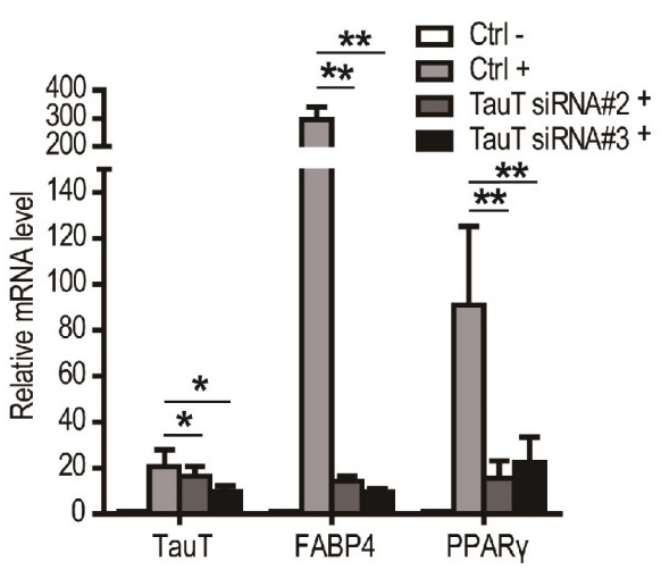

C

Ctrl

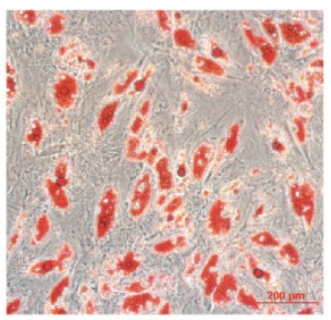

TauT-KD

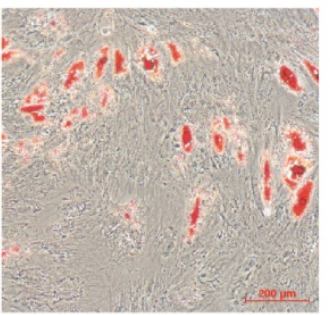

B

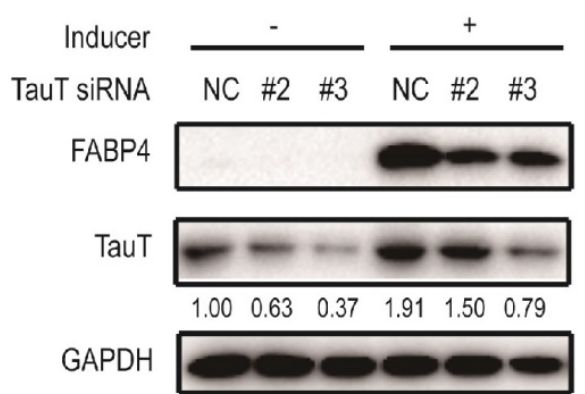

D

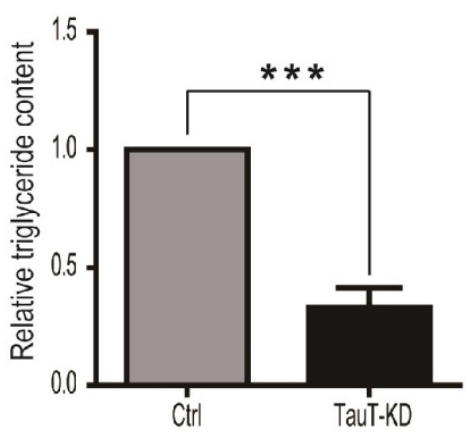

F

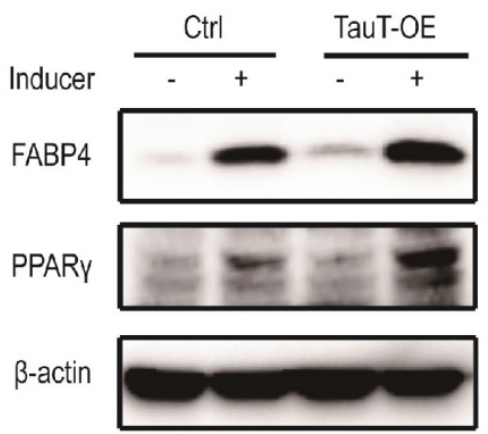

H

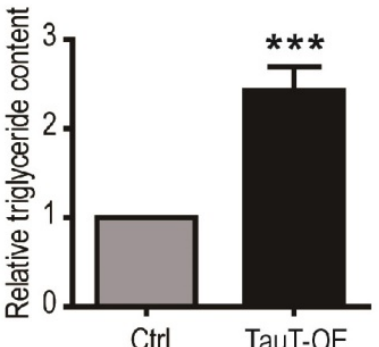

Figure 2. TauT promoted the adipogenic differentiation of hASCs. A. The mRNA expression of adipocyte differentiation markers in TauT silencing cells. hASCs transfected with the control or TauT siRNAs and subsequently treated with or without adipogenic inducer for 3 days and cells were harvested for RT-PCR $(n=3, * p<0.05$, ** $<<0.01)$. B. The protein expression of FABP4 in TauT silencing cells in the presence or absence of inducer. Cells treated with or without adipogenic inducer for 3 days and cells were harvested for western blot. The numbers below FABP4 bands were relatively grayscale values qualified by Image J compared to GAPDH. C. The intracellular lipid accumulation in TauT silencing cells. hASCs transfected with the control or TauT siRNAs every 3 days and subsequently induced adipogenic differentiation. Oil Red O staining was performed at day 9. D. The intracellular triglyceride content in TauT silencing cells. E. The mRNA expression of adipocyte differentiation markers in TauT overexpression cells. hASCs were treated with or without adipogenic inducer for 3 days and cells were harvested for RT-PCR $(n=3, *<<0.05$, ** $<<0.01)$. F. The protein expression of FABP4 in TauT overexpression cells in the presence or absence of inducer. hASCs were treated with or without adipogenic inducer for 3 days and cells were harvested for western blot. G. The intracellular lipid accumulation in TauT overexpression cells. hASCs transfected with the control or TauT overexpression plasmid and subsequently induced adipogenic differentiation. Oil Red O staining was performed at day 9 . H. The intracellular triglyceride content in TauT overexpression cells. All Western blots were independently repeated three times, and RT-PCR data are presented as the means $\pm \mathrm{SD}$. 
A

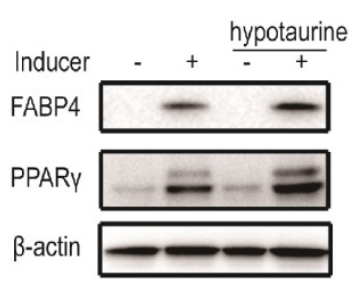

B

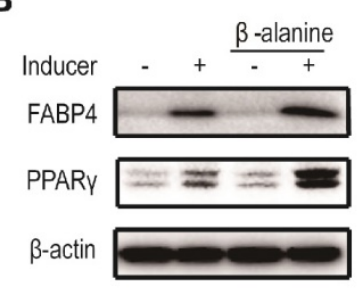

C

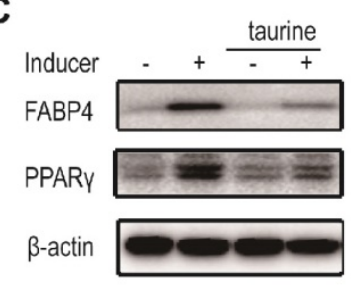

D

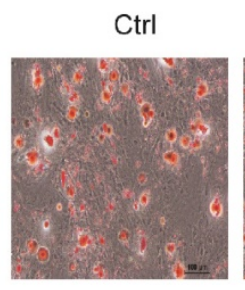

hypotaurine

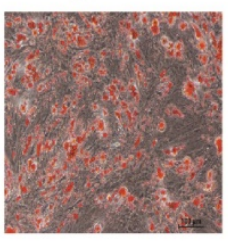

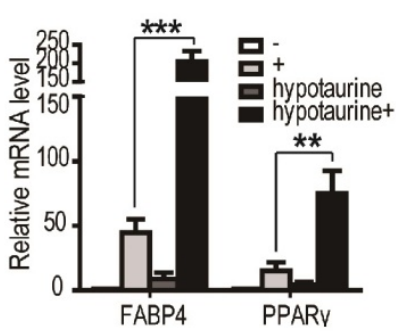
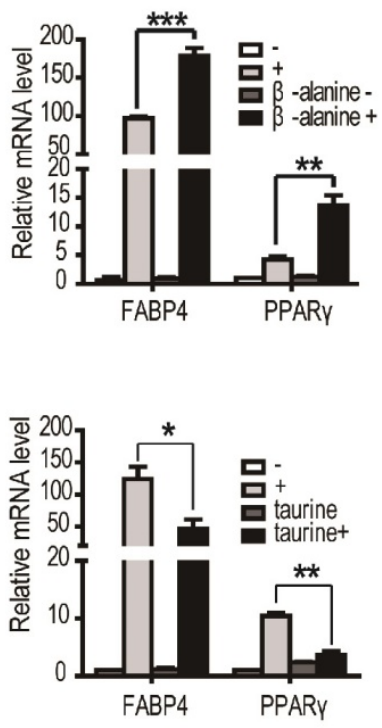
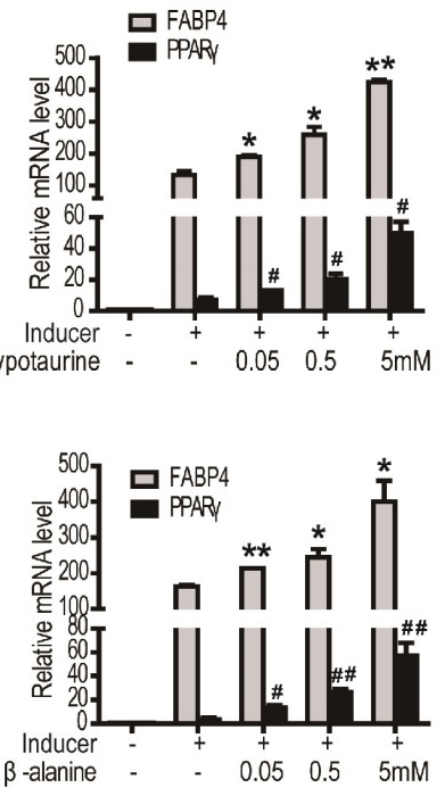

$\beta$-alanine

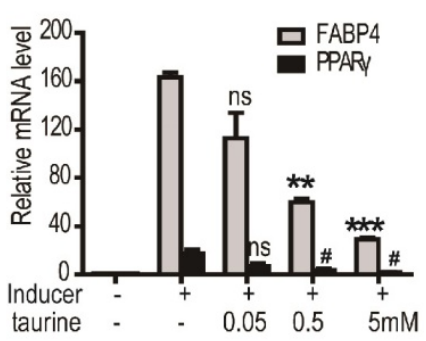

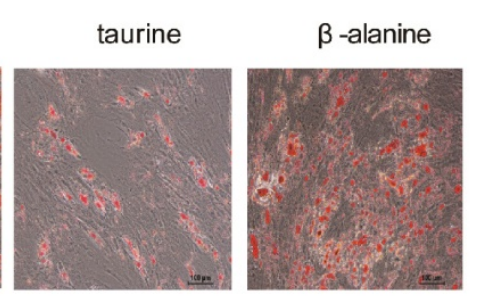

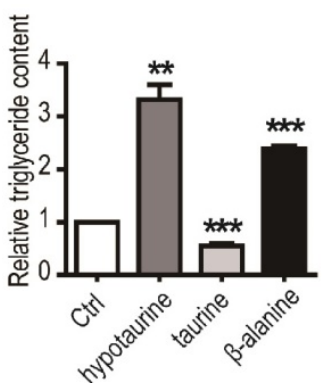

Figure 3. Hypotaurine and $\beta$-alanine promoted the adipogenic differentiation of hASCs, while taurine inhibited. A. The expression of adipocyte differentiation markers in the presence or absence of hypotaurine. hASCs were treated with or without adipogenic inducer and hypotaurine for 3 days and cells were harvested for western blot and RT-PCR $\left(n=3, * \mathrm{p}\right.$ or ${ }^{\mathrm{p}}<0.05$, ** $\left.<0.01, * * * \mathrm{p}<0.001\right)$. B. The expression of adipocyte differentiation markers in the presence or absence of $\beta$-alanine. hASCs were treated with or without adipogenic inducer and $\beta$-alanine for 3 days and cells were harvested for western blot and RT-PCR ( $n=3$, ${ }^{*} \mathrm{p}$ or ${ }^{\#} \mathrm{p}<0.05,{ }^{* *} \mathrm{p}$ or $\left.\mathrm{m}^{\mathrm{p}}<0.01, * * * \mathrm{p}<0.001\right)$. C. The expression of adipocyte differentiation markers in the presence or absence of taurine. hASCs were treated with or without adipogenic inducer and taurine for 3 days and cells were harvested for western blot and RT-PCR $(n=3, n s p>0.05$, *p or \#p $<0.05$, ** $p<0.01$, **** $<<0.001)$. D. The intracellular lipid accumulation and triglyceride content in the presence or absence of hypotaurine, taurine or $\beta$-alanine. hASCs were treated with hypotaurine $(5 \mathrm{mM}), \beta$-alanine $(5 \mathrm{mM})$ or taurine $(5 \mathrm{mM})$ and induced adipogenic differentiation. Oil Red $\mathrm{O}$ staining was performed at day 9 . All Western blots were independently repeated three times, and RT-PCR data are presented as the means \pm SD.

\section{The inhibitory effect of TauT knockdown on adipogenic differentiation was reversed by hypotaurine and $\beta$-alanine, but not taurine}

To further assess whether the role of TauT on adipognesis is related to its ability to transport the amino acids, we knocked down TauT of hASCs in the presence exogenous of hypotaurine, $\beta$-alanine, or taurine. As shown in Figure 4A, downregulation of FABP4 and PPARY in induced hASCs by TauT knockdown were reversed significantly by hypotaurine and $\beta$-alanine, but not taurine. We also assessed the formation of adipocytes by Oil Red $\mathrm{O}$ staining and triglyceride quantification after the induction was continued for 9 days. While the Oil Red $\mathrm{O}$ staining was markedly reduced by TauT knockdown (Figure 4B, panel 3), the presence of hypotaurine and $\beta$-alanine significantly alleviated the reduction (Figure 4B, panel 4 \& 6). However, addition 
of taurine to the culture did not rescue the decrease of Oil Red O staining (Figure 4B, panel 5). Similarly, the decrease of total triglyceride by TauT knockdown was rescued to a large extent by hypotaurine and $\beta$-alanine, but not taurine (Figure 4C). These results indicated that the taurine transporter affects adipogenesis at least partially through transporting these amino acids.

\section{TauT regulated the adipogenic differentiation through preventing the downregulation of $W n t / \beta$-catenin signaling}

The Wnt/ $\beta$-catenin signaling pathway plays a critical role in maintaining the stemness of various progenitor cells including hASCs $\underline{15}$. It has been shown

A

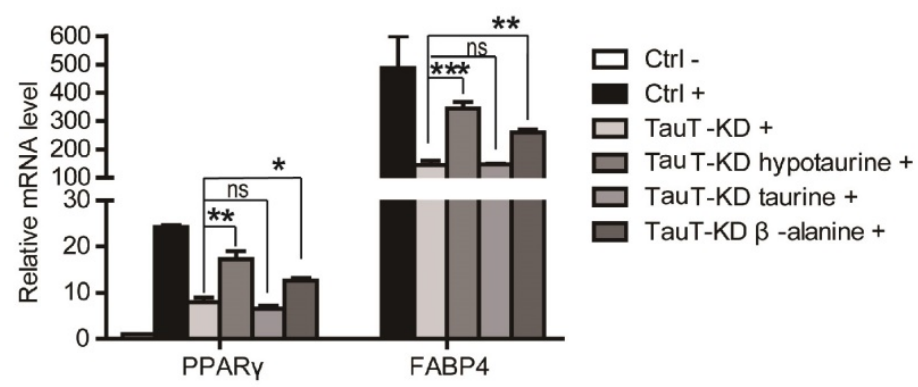

B

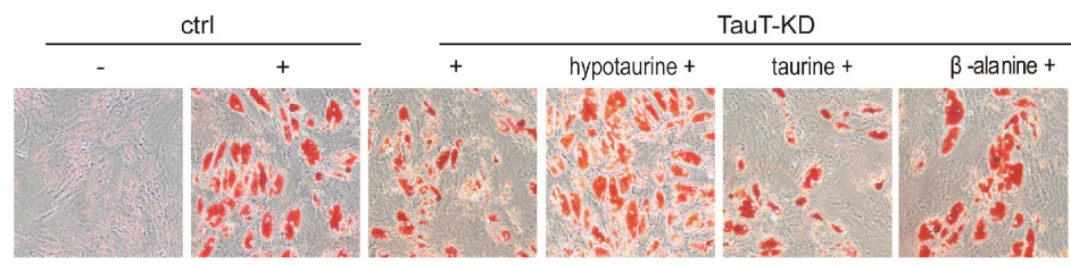

C

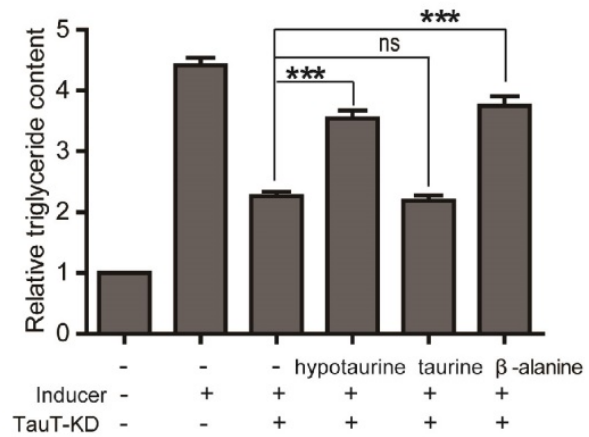

Figure 4. The inhibitory effect of TauT knockdown on hASCs adipogenic differentiation could be reversed by hypotaurine and $\beta$-alanine, but not taurine. A. The mRNA expression of adipocyte differentiation markers in TauT knockdown cells treated with hypotaurine, taurine or $\beta$-alanine. hASCs transfected with the control or TauT siRNAs and subsequently induced adipogenic differentiation. Meanwhile, $5 \mathrm{mM}$ hypotaurine, taurine or $\beta$-alanine was added. After 3 days, cells were harvested for RT-PCR $\left(n=3,{ }^{n s} p>0.05,{ }^{*} p<0.05,{ }^{* *} p<0.01,{ }^{* * *} p<0.001\right)$. B \& C. The intracellular lipid accumulation and triglyceride content in TauT knockdown cells treated with hypotaurine, taurine or $\beta$-alanine. hASCs transfected with the control or TauT siRNAs every 3 days and subsequently induced adipogenic differentiation and $5 \mathrm{mM}$ hypotaurine, taurine or $\beta$-alanine was added. Oil Red $O$ staining was performed at day $9(B)$. Then the content of triglyceride was quantified (C). that down -regulation of the pathway is required for the adipogenic differentiation of hASCs and activation of Wnt receptor prevented the differentiation $\underline{16}$. As shown in Figure 5A, adipogenic differentiation of hASCs led to reduced expression of $\beta$-catenin. TauTspecific siRNAs prevented the $\beta$-catenin reduction and the expression of differentiation marker (Figure 5B). Noteworthily, both hypotaurine and $\beta$-alanine reduced the level of $\beta$-catenin dose-dependently (Figure 5C\&5D), whereas taurine prevented the reduction of $\beta$-catenin (Figure 5E), recapitulating their effects on adipogenesis. Moreover, hypotaurine and $\beta$-alanine, but not taurine, abolished to a large extent the increase of $\beta$-catenin in TauT-knockdown hASCs induced to differentiate (Figure 5F). TWS119, an activator of $\mathrm{Wnt} / \beta$-catenin through inhibiting GSK3 $\beta$, prevented adipogenic differentiation promoted by hypotaurine and $\beta$-alanine (Figure 5G). Moreover, exposing to TWS119 also abolished the pro-differentiation effect of overexpressing TauT in hASCs (Figure $\mathbf{5 H}$ ). Taken together, these results indicated that TauT participated the downregulation of Wnt/ $\beta$-catenin signaling pathway during adipogenic differentiation, and the effect is at least partially mediated by the amino acids it transports.

\section{Discussion}

It has been shown that the CCAAT/Enhancer- binding Protein (C/EBP) family proteins C/EBP $\beta$ and $\delta$ are among the initially upregulated transcription factors after the progenitor cells were induced to differentiation toward adipocytes $\underline{17}$. The subsequent increases of C/EBPa and peroxisome proliferator- activated receptor $\mathrm{Y}$ (PPARY) are responsible for the induction of a variety of changes in gene expression during adipocye formation, including the expression of FABP4 and lipoprotein lipase 18 . These transcription factors also act to suppress the Wnt/ $\beta$-catenin signaling, which is required for adipogenic differentiation 19 . We found in the present study that taurine transporter was markedly increased during adipogenesis, whereas knockdown of TauT impeded adipocyte 
formation, indicating that the transporter promotes adipogenic differentiation. We also found that TauT expression is elevated in adipose tissues from mice fed with high fat diet or harboring mutations in lepin or leptin receptor. Consistent with the result, it has been shown that TauT-knockout mice exhibited lower body weight and abdominal fat mass than wild-type mice 20 . Since both C/EBP-like family and PPARY are capable of increasing TauT expression in various cells $\underline{21}, 22$, it is conceivable that TauT might act as a positive feedback to amplify the differentiating signal, and serve as a target for intervening adipogenesis. It is worth noting that TauT may transport multiple substrates including taurine, hypotaurine, $\gamma$-aminobutyric acid and $\beta$-alanine into cells, and meanwhile output ions from the cells $\underline{23}$. However, only high doses of hypotaurine and $\beta$-alanine promoted adipogenesis and reversed the inhibitory effects of TauT knockdown on hASCs differentiation, suggesting that these amino acids are required for adipocyte formation or they can enhance the functional activity of TauT. Interestingly, it was demonstrated recently that taurine supplementation attenuated adipogenesis and inhibited TauT expression in animal experiments 24 . In hASCs, we also found taurine reduced the expression of TauT dose-dependently (data not shown). Thus, it is interesting to know whether hypotaurine and $\beta$-alanine could affect TauT expression. It is conceivable that high dose taurine inhibits adipogenic differentiation due to the reduction of cross cell membrane gradient of taurine and reduction of TauT.

Although taurine is not a building block of protein, it is found in millimolar concentration in mammalian tissue such as skeletal and heart muscles, and can be absorbed from dietary and synthesized from cysteine ${ }^{25}$. Interestingly, while the intracellular concentration of taurine is in the ranges of $10-20 \mathrm{mM}$, its plasma level is only about one 500th $\underline{26}$. The importance of taurine transportation was also clearly illustrated by the finding that the taurine levels in skeletal and heart muscle of TauT-deficient mice decreased by about $98 \%$ compared with that of controls $\underline{20}$. Noteworthily, in addition to be essential for the function of skeletal muscle, the retina, the cardiovascular and central nervous system 27 , taurine supplemented in drink water had significant anti-obesity effects in animals fed with high fat diet and in genetic obese mice $24, \underline{25}$. Epidemiological studies also found that urinary taurine content was inversely related to BMI and cadiovascular risks 28 . Our finding that high concentration of taurine effectively blocked the adipocyte formation provided an attractive rationale for the anti-obesity activity, and may account for the change of macrophage activity and production of inflammatory cytokines in adipose tissues. This is also consistent with the report that macrophages-derived taurine chloramine inhibited the differentiation of preadipocytes into adipocytes and modulated the expression of adipokines in adipocytes 29 .
A

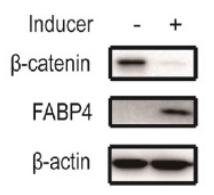

C

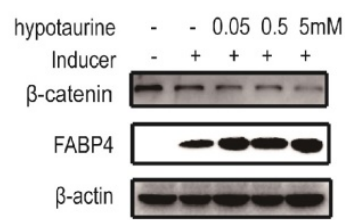

E

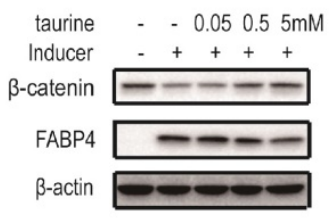

G

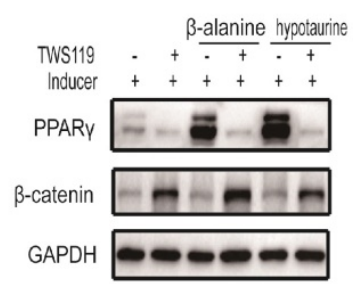

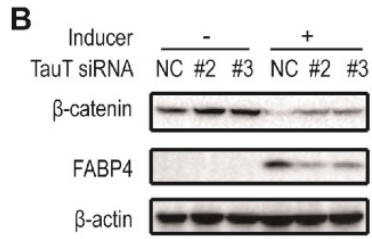

D

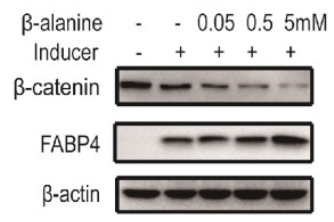

$\mathbf{F}$

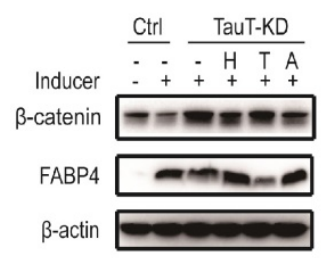

H

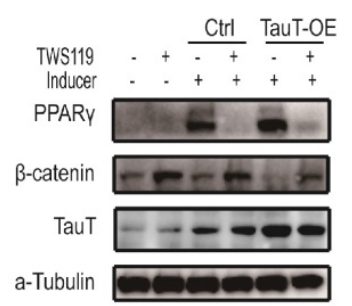

Figure 5. TauT regulated the adipogenic differentiation through preventing the downregulation of $W n t / \beta$-catenin signaling. A. The expression of $\beta$-catenin in the presence or absence of inducer. B. The expression of $\beta$-catenin in TauT silencing cells in the presence or absence of inducer. hASCs transfected with the control or TauT siRNAs and subsequently treated with or without adipogenic inducer for 3 days. C. The expression of $\beta$-catenin under inducer and hypotaurine dose points treatment. hASCs were treated with adipogenic inducer and the indicated dose of hypotaurine for 3 days. $\mathbf{D}$. The expression of $\beta$-catenin under inducer and $\beta$-alanine dose points treatment. hASCs were treated with adipogenic inducer and the indicated dose of $\beta$-alanine for 3 days. E. The expression of $\beta$-catenin under inducer and taurine dose points treatment. hASCs were treated with adipogenic inducer and the indicated dose of taurine for 3 days. F. The expression of $\beta$-catenin in TauT knockdown cells treated with hypotaurine, taurine or $\beta$-alanine. hASCs transfected with the control or TauT siRNAs and subsequently induced adipogenic differentiation. Meanwhile, $5 \mathrm{mM}$ hypotaurine, taurine or $\beta$-alanine was added for 3 days. FABP4 were detected as marker protein of adipogenic differentiation. G. The expression of PPARY in hypotaurine or $\beta$-alanine treated cells in the presence or absence of GSK3 $\beta$ inhibitor. hASCs were treated with hypotaurine or $\beta$-alanine and adipogenic inducer for 3 days, 50nM TWS1 19 was added in the meantime. $\mathbf{H}$. The expression of PPARY in TauT overexpression cells in the presence or absence of GSK3 $\beta$ inhibitor. The control and TauT overexpression hASCs were treated with or without adipogenic inducer and GSK3 $\beta$ inhibitor for 3 days. All Western blots were independently repeated three times. 
In summary, we have started to analyze the differentiation mechanisms of hASCs, a rich source of potential stem cells that could differentiate into many different tissue cells. Our data showed the Taurine transporter played an important role in the differentiation of hASCs into adipocytes. Intriguingly, while TauT substrates hypotaurine and $\beta$-alanine promoted adipogenesis, taurine inhibited effectively adipocyte formation. They appeared act in hASCs by promoting and inhibiting $\beta$-catenin reduction respectively, which was essential for hASCs differentiation. Thus, TauT is a potential target for preventive or therapeutic intervention of disorders such as obesity and fatty liver. Noteworthily, the taurine transporter expression has also been linked to diabetes, cardiovascular and neurological diseases ${ }^{30}-32$. It is conceivable that the different activities of its substrates might provide novel clues to understand and treat these diseases.

\section{Supplementary Material}

Supplementary figures and tables. http://www.ijbs.com/v15p1104s1.xls

\section{Acknowledgement}

This study was supported by PUMC Youth Fund (2017310041), the Non-profit Central Research Institute Fund of Chinese Academy of Medical Sciences (2017NL31002), National Natural Science Foundation of China (81572378), CAMS Initiative for Innovative Medicine (CAMS-I2M, 2016-I2M-1005), the Special Research Fund for Central Universities, Peking Union Medical College (2016ZX310194), the Fund from Jiangsu Provincial Science and Technology Department (BM2016006), and the Fund from Suzhou Municipal Science and Technology Bureau (SZS201716).

\section{Competing Interests}

The authors have declared that no competing interest exists.

\section{References}

1. Trayhurn P, Wang B, Wood IS. Hypoxia and the endocrine and signalling role of white adipose tissue. Arch Physiol Biochem. 2008; 114: 267-76.

2. Alvarez-Llamas G, Szalowska E, de Vries MP, et al. Characterization of the human visceral adipose tissue secretome. Mol Cell Proteomics. 2007; 6: 589-600.

3. Rosen ED, Spiegelman BM. Adipocytes as regulators of energy balance and glucose homeostasis. Nature. 2006; 444: 847-53.

4. Mercer JG, Hoggard N, Williams LM, et al. Coexpression of leptin receptor and preproneuropeptide $\mathrm{Y}$ mRNA in arcuate nucleus of mouse hypothalamus. J Neuroendocrinol. 1996; 8: 733-5.

5. Munzberg H, Morrison CD. Structure, production and signaling of leptin. Metabolism. 2015; 64: 13-23.

6. Caballero B. The global epidemic of obesity: an overview. Epidemiol Rev. 2007; 29: 1-5.

7. Jung UJ, Choi MS. Obesity and its metabolic complications: the role of adipokines and the relationship between obesity, inflammation, insulin resistance, dyslipidemia and nonalcoholic fatty liver disease. Int J Mol Sci. 2014; 15: 6184-223.
8. Riondino S, Roselli M, Palmirotta R, et al. Obesity and colorectal cancer: role of adipokines in tumor initiation and progression. World J Gastroenterol. 2014; 20: 5177-90.

9. Unamuno X, Gomez-Ambrosi J, Rodriguez A, et al. Adipokine dysregulation and adipose tissue inflammation in human obesity. Eur J Clin Invest. 2018; 48: e12997.

10. Serena C, Keiran N, Ceperuelo-Mallafre V, et al. Obesity and Type 2 Diabetes Alters the Immune Properties of Human Adipose Derived Stem Cells. Stem Cells. 2016; 34: 2559-73.

11. Ding F, Qiu J, Li Q, et al. Effects of rosiglitazone on proliferation and differentiation of duck preadipocytes. In Vitro Cell Dev Biol Anim. 2016; 52: 174-81.

12. Garris DR. Obese (ob/ob) and diabetes $(\mathrm{db} / \mathrm{db})$ mutations: two factors modulating brain and peripheral tissue accumulation of estradiol in C57BL/KsJ mice. Brain Res. 1987; 432: 153-7.

13. Duggal $P$, Guo $X$, Haque $R$, et al. A mutation in the leptin receptor is associated with Entamoeba histolytica infection in children. J Clin Invest. 2011; 121: 1191-8

14. Jhiang SM, Fithian L, Smanik $\mathrm{P}$, et al. Cloning of the human taurine transporter and characterization of taurine uptake in thyroid cells. FEBS Lett. 1993; 318: 139-44.

15. Li HX, Luo X, Liu RX, et al. Roles of Wnt/beta-catenin signaling in adipogenic differentiation potential of adipose-derived mesenchymal stem cells. Mol Cell Endocrinol. 2008; 291: 116-24.

16. Moldes M, Zuo Y, Morrison RF, et al. Peroxisome-proliferator-activated receptor gamma suppresses $\mathrm{Wnt} /$ beta-catenin signalling during adipogenesis. Biochem J. 2003; 376: 607-13.

17. Cao Z, Umek RM, McKnight SL. Regulated expression of three C/EBP isoforms during adipose conversion of 3T3-L1 cells. Genes Dev. 1991; 5: 1538-52.

18. Wu Z, Rosen ED, Brun $\mathrm{R}$, et al. Cross-regulation of C/EBP alpha and PPAR gamma controls the transcriptional pathway of adipogenesis and insulin sensitivity. Mol Cell. 1999; 3: 151-8.

19. Farmer SR. Regulation of PPARgamma activity during adipogenesis. Int J Obes (Lond). 2005; 29 Suppl 1: S13-6.

20. Ito $\mathrm{T}$, Yoshikawa $\mathrm{N}$, Ito $\mathrm{H}$, et al. Impact of taurine depletion on glucose control and insulin secretion in mice. J Pharmacol Sci. 2015; 129: 59-64.

21. Chen Z, He P, Ding X, et al. PPARgamma stimulates expression of L-type amino acid and taurine transporters in human placentas: the evidence of PPARgamma regulating fetal growth. Sci Rep. 2015; 5: 12650.

22. Grewal S, Defamie N, Zhang X, et al. SNAT2 amino acid transporter is regulated by amino acids of the SLC6 gamma-aminobutyric acid transporter subfamily in neocortical neurons and may play no role in delivering glutamine for glutamatergic transmission. J Biol Chem. 2009; 284: 11224-36.

23. Yahara $\mathrm{T}$, Tachikawa $\mathrm{M}$, Akanuma $\mathrm{S}$, et al. Amino acid residues involved in the substrate specificity of TauT/SLC6A6 for taurine and gammaaminobutyric acid. Biol Pharm Bull. 2014; 37: 817-25.

24. Kim KS, Jang MJ, Fang S, et al. Anti-obesity effect of taurine through inhibition of adipogenesis in white fat tissue but not in brown fat tissue in a high-fat diet-induced obese mouse model. Amino Acids. 2019; 51: 245-54

25. Murakami S. The physiological and pathophysiological roles of taurine in adipose tissue in relation to obesity. Life Sci. 2017; 186: 80-6.

26. Learn DB, Fried VA, Thomas EL. Taurine and hypotaurine content of human leukocytes. J Leukoc Biol. 1990; 48: 174-82.

27. Shiohira S, Komatsu $M$, Okazaki $M$, et al. Effect of Taurine on Hemodiafiltration in Patients With Chronic Heart Failure. Ther Apher Dial. 2016; 20: 20-6

28. Sagara M, Murakami S, Mizushima S, et al. Taurine in 24-h Urine Samples Is Inversely Related to Cardiovascular Risks of Middle Aged Subjects in 50 Populations of the World. Adv Exp Med Biol. 2015; 803: 623-36.

29. Marcinkiewicz J, Grabowska A, Bereta J, et al. Taurine chloramine, a product of activated neutrophils, inhibits in vitro the generation of nitric oxide and other macrophage inflammatory mediators. J Leukoc Biol. 1995; 58: 667-74.

30. Napoli Z, Seghieri G, Bianchi L, et al. Taurine Transporter Gene Expression in Mononuclear Blood Cells of Type 1 Diabetes Patients. J Diabetes Res. 2016; 2016: 7313162

31. Ito T, Kimura Y, Uozumi Y, et al. Taurine depletion caused by knocking out the taurine transporter gene leads to cardiomyopathy with cardiac atrophy. J Mol Cell Cardiol. 2008; 44: 927-37.

32. Warskulat U, Heller-Stilb B, Oermann E, et al. Phenotype of the taurine transporter knockout mouse. Methods Enzymol. 2007; 428: 439-58. 